\title{
Análisis de rastros en el desplazamiento de cangrejos ermitaño terrestres: una ventaja al usar conchas ligeras
}

\author{
Oscar Larrañaga, Karla Kruesi
}

\begin{abstract}
Oscar Larrañaga
Karla Kruesi

kruesi@ciencias.unam.mx

Laboratorio de Ecofisiología, Facultad de Ciencias, Universidad Nacional Autónoma de México, Avenida Universidad 3000, Ciudad Universitaria, Coyoacán, 04510, Ciudad de México, México.
\end{abstract}

BOL. SOC. GEOL. MEX. 2018

VOL. 70 NO. 2

P. $369-377$

http://dx.doi.org/10.18268/BSGM2018v70n2a7

\section{RESUMEN}

Para los cangrejos ermitaño, uno de los recursos más importantes es la concha de gasterópodos que portan a lo largo de su vida. Esta concha les proporciona diferentes beneficios como protección, refugio y evita la desecación. En los ermitaños semi-terrestres (Familia Coenobitidae) se presenta una conducta a través de la cual remodelan sus conchas desde el interior, incrementando el volumen interno y haciéndolas más ligeras. En este estudio se evaluaron algunas ventajas en términos de locomoción que estos organismos adquieren como resultado de este proceso de remodelación. Para esto se recolectaron cangrejos ermitaño terrestres de la especie Coenobita compressus en Playa Troncones, Guerrero. Se realizaron experimentos de locomoción en los que los cangrejos caminaron en arena húmeda ocupando conchas nuevas sin remodelar (pesadas), y conchas remodeladas (ligeras) con el fin de medir su desempeño. Se tomaron medidas de profundidad y tipo de huellas, además de la velocidad de la caminata de cada cangrejo.

El resultado fue que los cangrejos que utilizaban conchas remodeladas presentaban un menor arrastre, huellas de la concha menos profundas y alcanzaron una mayor velocidad. Por lo tanto, se lograron demostrar ventajas obtenidas tras remodelar la concha desde un punto de vista locomotriz. Este estudio además nos muestra una aplicación de neo-icnología como herramienta en estudios del área ecológica y biológica.

Palabras clave: Conchas remodeladas, conchas sin remodelar, locomoción, huellas, profundidad, velocidad.

\section{ABSTRACT}

For the hermit crabs, one of the most important resources is the shell of gastropod they carry along their life. This shell gives them different benefits like protection, refuge and prevents desiccation. The semi-terrestrial hermit crabs (Family Coenobitidae) perform a behavioral remodeling process from the inside of the gastropod shell, increasing its internal volume and making them lighter. In this study the advantages provided by remodeling a shell were evaluated with a locomotion approach. For this, terrestrial hermit crabs from Coenobita compressus species were collected in Playa Troncones, Guerrero. Locomotion experiments were performed where the crabs walked on wet sand occupying remodeled and non-remodeled shells with the purpose of measure their performance. Depth measurements and type of imprint were taken; also the walking velocity of each crab was measured. As a result we obtained that the hermit crabs occupying remodeled shells had a lower drag, shallower shell traces and they reached a greater speed. Therefore, the benefits of using a remodeled shell were demonstrated from a locomotive point of view. Besides, this study showed an application of the neo-ichnology as an implement in ecological and biological studies.

Keywords: Remodeled shells, non-remodeled shells, locomotion, imprints, depth, walking velocity. 


\section{Introducción}

Los cangrejos ermitaño utilizan conchas de gasterópodos como un recurso que principalmente los protege ante el riesgo de depredación y desecación, ya que ésta guarda una reserva de agua y sal, lo que les permite alejarse para forrajear a distancias largas, lejos de una fuente de agua, o desplazarse varios kilómetros para liberar a sus larvas en el océano (Herreid, 1969; de Wilde, 1973; Angel, 2000); por esto, las conchas pueden considerarse como uno de sus recursos más importantes para la supervivencia. Al desplazarse, estos crustáceos cargan la estructura que les sirve de refugio, lo que representa desafios en términos energéticos y de locomoción (Herreid y Full, 1986; Osorno et al., 1998). La arena o suelos fangosos de esteros son el substrato más común sobre el que se desplazan los cangrejos ermitaño de hábitos semi-terrestres de la familia Coenobitidae Dana, 1851 (Barnes, 1997). Con excepción del género Birgus Leach, 1815 que no utiliza una concha en el estadio adulto, los ermitaños terrestres difieren a todas las especies acuáticas al presentar un mecanismo a través del cual desgastan o remodelan sus conchas química y mecánicamente desde el interior, reduciendo así el peso de la concha mientras aumentan su volumen interno (Laidre et al., 2012; Vermeij, 2012). Este mecanismo de remodelación de un refugio que es móvil, les da ventajas a los cangrejos durante la locomoción en términos del gasto energético, además que incrementa el espacio para el reservorio de agua en el interior de la concha (Laidre, 2014). La locomoción en un medio granular como la arena, representa un reto importante para los organismos que desempeñan actividades ecológicamente relevantes en este sustrato, ya que no es un medio sólido (Herreid y Full, 1986; Mazouchova et al., 2010). En este estudio se realizaron diferentes pruebas de locomoción en cangrejos ermitaño terrestres de la especie Coenobita compressus MilneEdwards, 1834, utilizando algunos métodos de neoicnología, como el análisis de rastros, con el fin de demostrar que el mecanismo de remodelación de conchas que presentan estos anomuros como resultado de su evolución, representa ventajas en su desempeño de desplazamiento. La hipótesis de este estudio predice que las conchas que no han sido remodeladas son más pesadas, y por lo tanto, los ermitaños no podrían cargarlas de manera constante mientras se desplazan, dejando sus rastros sobre el arena. Adicionalmente, el arrastre que las conchas generan resultará en una disminución de la velocidad de caminata, en comparación con la que los ermitaños pueden alcanzar cuando se desplazan con sus conchas remodeladas.

\section{Métodos}

\subsection{MUESTREO YMANTENIMIENTO DE ORGANISMOS EXPERIMENTALES}

En este estudio, la recolecta y los experimentos fueron realizados en Playa Troncones, localizada en la zona noroeste del estado de Guerrero, en el municipio de La Unión (1746’48.68”N, $101^{\circ} 43^{\prime} 23.20^{\prime \prime O}$; Figura 1). Los cangrejos ermitaño (Coenobita compressus) fueron recolectados sobre la playa hasta obtener 30 ejemplares. La recolección se llevó a cabo durante la noche, seleccionando a todos los cangrejos que ocupaban conchas del gasterópodo Nerita scabricosta Lamarck, 1815. El género Nerita Linnaeus, 1758 ha sido reportado frecuentemente por proveer las conchas de uso más común para los ermitaños terrestres en costas del Pacífico tropical (Abrams, 1978), y específicamente también en costas mexicanas (Guillén y Osorno, 1993). Adicionalmente se recolectó arena (aproximadamente $4 \mathrm{~kg}$ ) en el sitio donde los ermitaños fueron capturados. Debido a que la arena tiene suspendidos numerosos objetos de basura orgánica que podrían modificar la impresión de huellas, la limpiamos con enjuagues de agua dulce, para después dejarla secar al sol y tamizarla. 


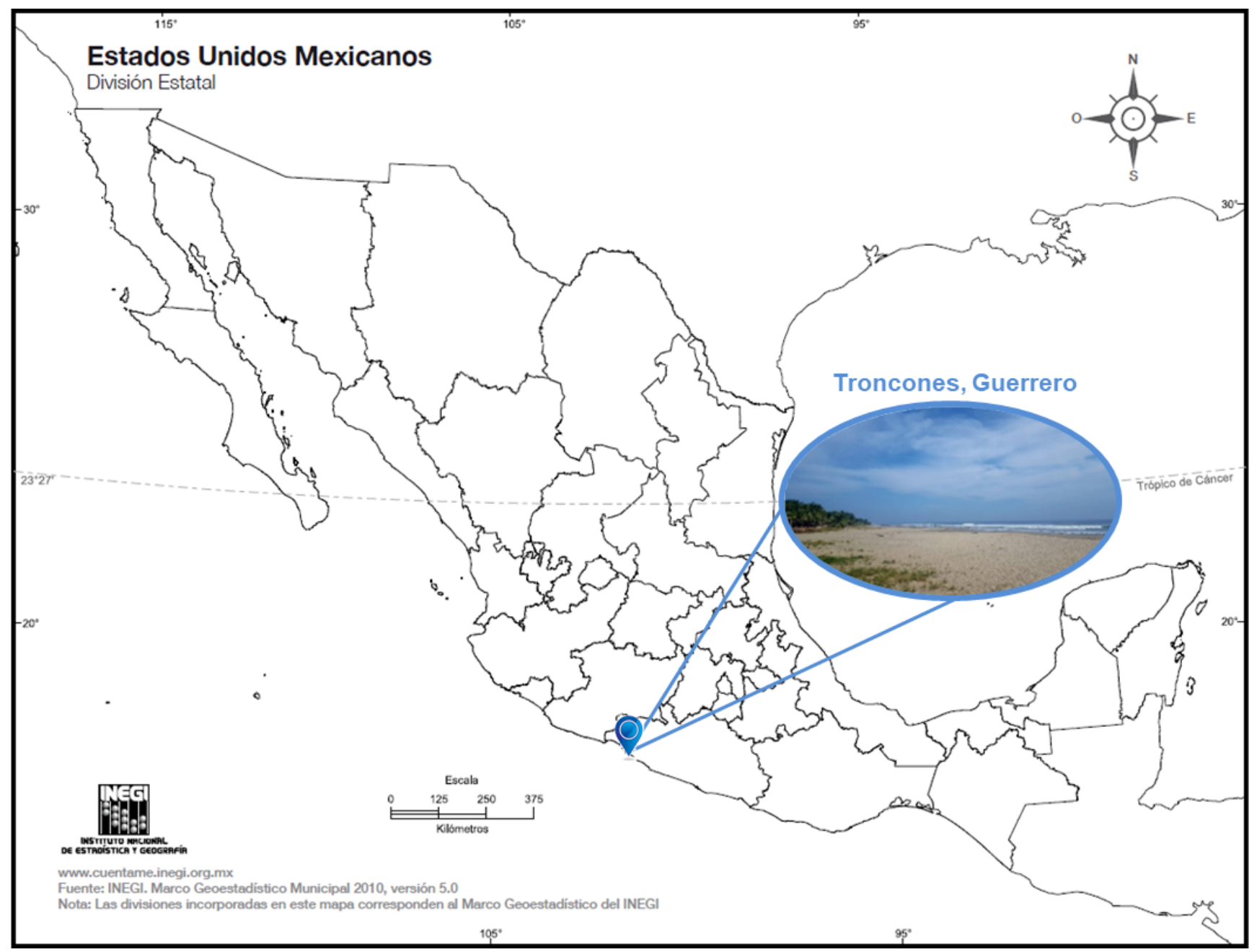

Figura 1 Mapa donde se muestra la zona de estudio: Playa Troncones, Guerrero. Imagen modificada de la obtenida desde el portal del Instituto Nacional de Estadística y Geografía (INEGI, 2010).

Posterior a su captura, los ermitaños se mantuvieron individualmente dentro de un cilindro de PVC (diámetro $=3 \mathrm{~cm}$, altura $=4 \mathrm{~cm}$ ) marcado con el número de individuo correspondiente (asignado al azar del 1 - 30), y cubierto con tapas de malla en ambos extremos. Los cilindros se colocaron dentro de una tina con el fondo permanentemente húmedo, alternando entre agua de mar y agua dulce. Los organismos experimentales fueron alimentados diariamente con fruta durante el tiempo que permanecieron en captura. Después de las fases de experimentación y medición (peso corporal, g; balanza de precisión OHAUS, \pm $0.001 \mathrm{~g}$ ), los cangrejos fueron liberados en el sitio de su recolección.

\subsection{DISEÑO EXPERIMENTAL}

Se utilizó un terrario de acrílico transparente (largo $\mathrm{x}$ ancho $\mathrm{x}$ altura $=30 \times 30 \times 8 \mathrm{~cm}$ ), llenándolo uniformemente de arena limpia obtenida de la localidad de colecta hasta una altura de 4 $\mathrm{cm}$, inundada con una película uniforme de agua dulce (1 mm sobre la arena). Las condiciones de profundidad de la cama de arena y la cantidad de agua utilizadas fueron determinadas tras realizar varias pruebas visuales (variando ambos factores) de los rastros obtenidos con cangrejos de talla similar a los utilizados en este estudio de acuerdo con Davis et al. (2007). 

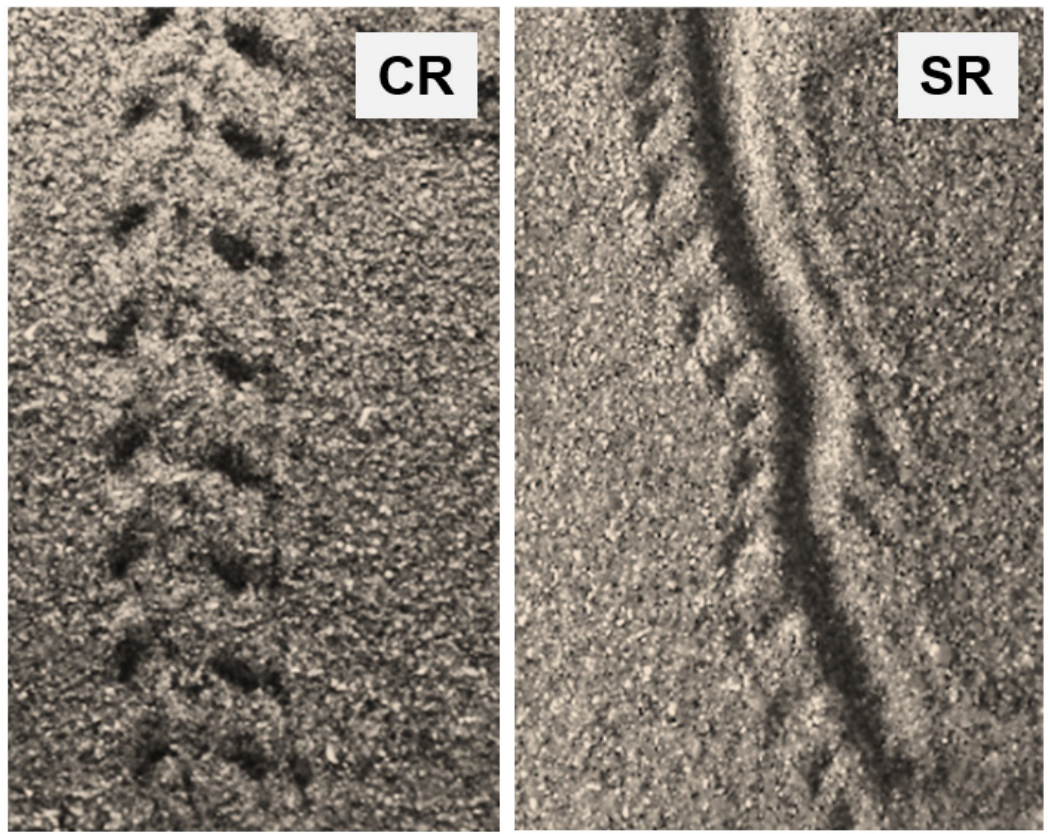

$1 \mathrm{~cm}$

Figura 2 Fotografías de los rastros de los cangrejos ermitaño sobre la arena utilizando una concha remodelada CR (concha modificada por los cangrejos ermitaño) y sin remodelar SR (concha sin modificar, obtenida de los gasterópodos que la producen). Se puede ver que el rastro al utilizar CR es discontinuo, mientras que, al utilizar SR, el rastro presenta una huella continua que es resultado de la fricción de la concha con el sustrato en la caminata del cangrejo ermitaño.

Sobre esta superficie se colocó a cada cangrejo en el centro, encerrándolo con un cilindro de PVC, donde permaneció durante 5 min como periodo de aclimatación. El desempeño de cada cangrejo fue probado en un ensayo diferente utilizando dos tipos de concha (remodelada, GR; y sin remodelar, SR). Los ensayos comenzaron después del periodo de aclimatación, al levantar el cilindro. Concluyeron cuando el ermitaño a prueba alcanzó alguna de las paredes del terrario, dejando un rastro de su desplazamiento marcado sobre la arena. Posteriormente se colocó nuevamente al cangrejo experimental en su cilindro de mantenimiento. Se extrajo el excedente de agua sobre el sedimento arenoso con un sifón (diámetro $=1 \mathrm{~cm}$ ) en una esquina del terrario, a la mayor distancia posible del rastro. Se finalizó la extracción de agua con un papel secante.

Cada ensayo fue grabado con una cámara de video (Nikon COOLPIX AW120), ajustada para filmar a una velocidad de 30 fps (por sus siglas en inglés, cuadros por segundo). Los rastros fueron fotografiados desde la misma altura con la cámara instalada dentro de una caja en condiciones de iluminación uniforme (2 lámparas largas MEGAMEX de luz fluorescente, 13 W). Sobre la arena en todos los ensayos se colocó una escala $(\mathrm{mm})$ como referencia para el registro de datos.

Cada organismo experimental fue utilizado dos veces en los dos tipos de conchas (GR y SR). En este estudio se controló experimentalmente cualquier sesgo en el desempeño de desplazamiento de los cangrejos con respecto al orden en que las conchas fueron utilizadas. Se seleccionaron 15 organismos al azar para evaluar su desempeño sobre la arena utilizando su concha original $(\mathrm{CR})$ y otros 15 individuos a los que se les asignó una concha de talla similar SR (longitud de apertura y diámetro; $\pm 2 \mathrm{~mm}$ ) como primera opción. Posteriormente se realizó un intercambio de concha a los ermitaños de manera que la segunda prueba experimental se llevó a cabo en la concha alternativa de talla similar. Las conchas SR fueron obtenidas de la colección de conchas del gasterópodo Nerita scabricosta 
A)

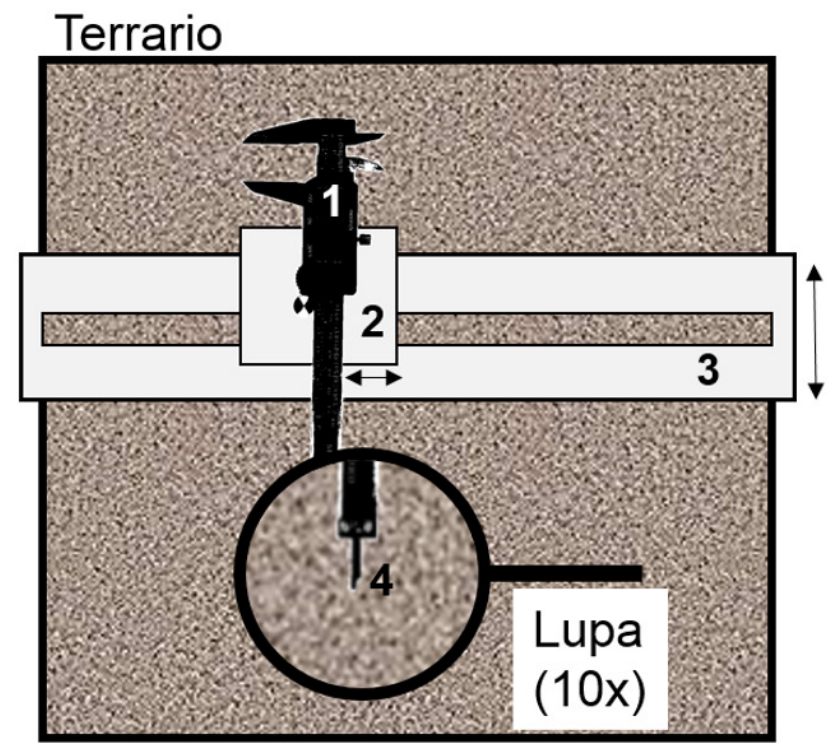

B)

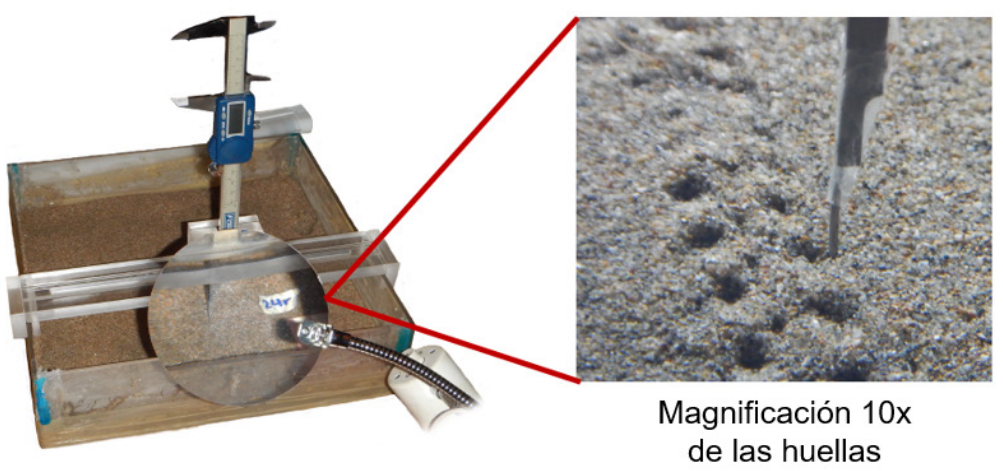

Figura 3 Descripción del sistema experimental que se utilizó en este estudio para registrar los rastros de los cangrejos ermitaño y estimar la profundidad de las huellas. A) El sistema (vista en planta) está compuesto por un terrario de acrílico con varios aditamentos para la medición de huellas: Calibrador digital (1) colocado en una guía de acrílico con una base para desplazarla de manera horizontal (2) y vertical (3). El calibrador fue complementado con una punta de aguja de insulina (4) que tocaba la superficie de la arena, visualmente detectable con una lupa de magnificación 10x. B) Fotografía del sistema experimental y de la imagen de las huellas magnificada (10x).

del Laboratorio de Ecofisiología, Universidad Nacional Autónoma de México.

\subsection{REGISTRO Y ANÁLISIS DE DATOS}

Se estimó y registró a través de las fotografías, de manera cuantitativa, la continuidad de la huella de la concha en el rastro que dejó cada cangrejo sobre la arena al desplazarse en CR y SR. Ésta fue registrada en cada caso como: continua (una línea continua a lo largo de todo el rastro, entre las huellas de los apéndices ambulatorios) o discontinua (una línea intermitente; Figura 2). Se llevó a cabo una prueba binomial que comparó las frecuencias de huellas continuas y discontinuas entre las dos muestras (CR y SR), contrastando la hipótesis nula de que la proporción de las huellas discontinuas sería mayor que 0.5.

Utilizando un calibrador digital con una aguja como punta que fue instalado en una guía de 
Tabla 1. Análisis cualitativos y cuantitativos de los rastros del desplazamiento de Coenobita compressus.

\begin{tabular}{|c|c|c|c|c|c|}
\hline \multicolumn{2}{|c|}{$\begin{array}{c}\text { Características de las } \\
\text { huellas }\end{array}$} & $\begin{array}{c}\text { CR } \\
\text { (remodeladas) }\end{array}$ & $\begin{array}{c}\text { SR } \\
\text { (sin remodelar) }\end{array}$ & $\begin{array}{c}\text { Prueba } \\
\text { estadística }\end{array}$ & $\begin{array}{l}\text { Resultado } \\
(\alpha=0.05)\end{array}$ \\
\hline \multirow[t]{2}{*}{ a } & Continuidad & $61 \%$ & $18 \%$ & Binomial & $\mathbf{C R} P=0.345$ \\
\hline & & discontinuas & discontinuas & $(28,0.5)$ & SR P $=0.001$ \\
\hline \multirow[t]{5}{*}{$\mathrm{b}$} & Profundidad & $(\mathrm{mm})$ & $(\mathrm{mm})$ & & \\
\hline & \multirow[t]{2}{*}{ Quelípedo } & Media $=0.571$ & Media $=0.567$ & $t$ de Student & $t=0.063$ \\
\hline & & $\mathrm{SD}=0.265$ & $\mathrm{SD}=0.309$ & pareada & $\mathrm{P}=0.018$ \\
\hline & \multirow[t]{2}{*}{ Concha } & Media $=0.441$ & Media $=0.723$ & $t$ de Student & $t=4.498$ \\
\hline & & $\mathrm{SD}=0.187$ & $\mathrm{SD}=0.322$ & pareada & $\mathrm{P}<0.001$ \\
\hline
\end{tabular}

Nota: La tabla muestra (a) datos de la proporción de huellas discontinuas que quedan en los rastros de los ermitaños al desplazarse utilizando conchas CR y SR, y (b) los valores de la profundidad de las huellas del quelípedo izquierdo y de la concha en el desplazamiento de los cangrejos utilizando los dos tipos de conchas.

acrílico que fue adaptado al terrario y una lupa de magnificación 10x (Figura 3), se estimó la profundidad de tres huellas consecutivas del quelípedo izquierdo desde su centro, así como tres medidas de la huella de la concha, en su porción central y paralelas horizontalmente a cada una de las huellas del quelípedo. El registro de huellas se midió iniciando a la distancia de un cuerpo y medio desde el punto de partida de cada individuo en cada uno de los ensayos. Se utilizó para cada medición la distancia de la punta de la aguja en la superficie de la arena donde no había rastro para calibrar el vernier en un valor de cero $(\mathrm{mm})$ y se trasladó de manera horizontal después sobre la guía de acrílico para medir la profundidad de cada par correspondiente de huellas (quelípedo izquierdo y concha). Se utilizó la mediana de los tres valores de profundidad de huellas (quelípedo izquierdo y concha) estimados. Posteriormente se comparó estadísticamente la variación de estos valores entre los grupos de datos de las huellas de los cangrejos que caminaron con conchas nuevas y con conchas remodeladas ( $t$ de Student pareada). Se llevó a cabo una prueba separada para comparar las huellas del quelípedo izquierdo y otra para las de la concha.

Adicionalmente, se estimó la velocidad de desplazamiento de los ermitaños (m/s) utilizando conchas GR y SR. Lo anterior se hizo analizando los videos obtenidos en cada ensayo con el programa de análisis de video y construcción de modelos Tracker (Brown, 2008). Éstos se compararon estadísticamente con una prueba de $t$ de Student pareada.

\section{Resultados}

La proporción de huellas discontinuas en los rastros del desplazamiento de los ermitaños utilizando conchas remodeladas (CR) aumentó a 61\% $(\mathrm{P}=0.345$; Tabla 1a), mientras que al caminar con una concha sin remodelar (SR), sólo el 18\% de las imágenes de los rastros del desplazamiento de los ermitaños sobre la arena fueron discontinuas (prueba binomial $\mathrm{P}=0.001$ ).

Adicionalmente, la profundidad de las huellas de las conchas en los rastros de los ermitaños que ocupaban una concha SR fue significativamente mayor que las huellas que quedaron marcadas en la arena cuando los organismos ocupaban conchas CR $(\mathrm{P}<0.001)$. Sin embargo, no se observaron diferencias en la profundidad de las huellas del quelípedo izquierdo al comparar aquellas de los cangrejos que caminaron portando los dos tipos de conchas $(\mathrm{P}=0.1764$; Tabla $1 \mathrm{~b})$.

Con respecto a la velocidad de desplazamiento, los resultados en este estudio demostraron que existen diferencias significativas entre los grupos de cangrejos cuando utilizaron cada uno de los tipos de 


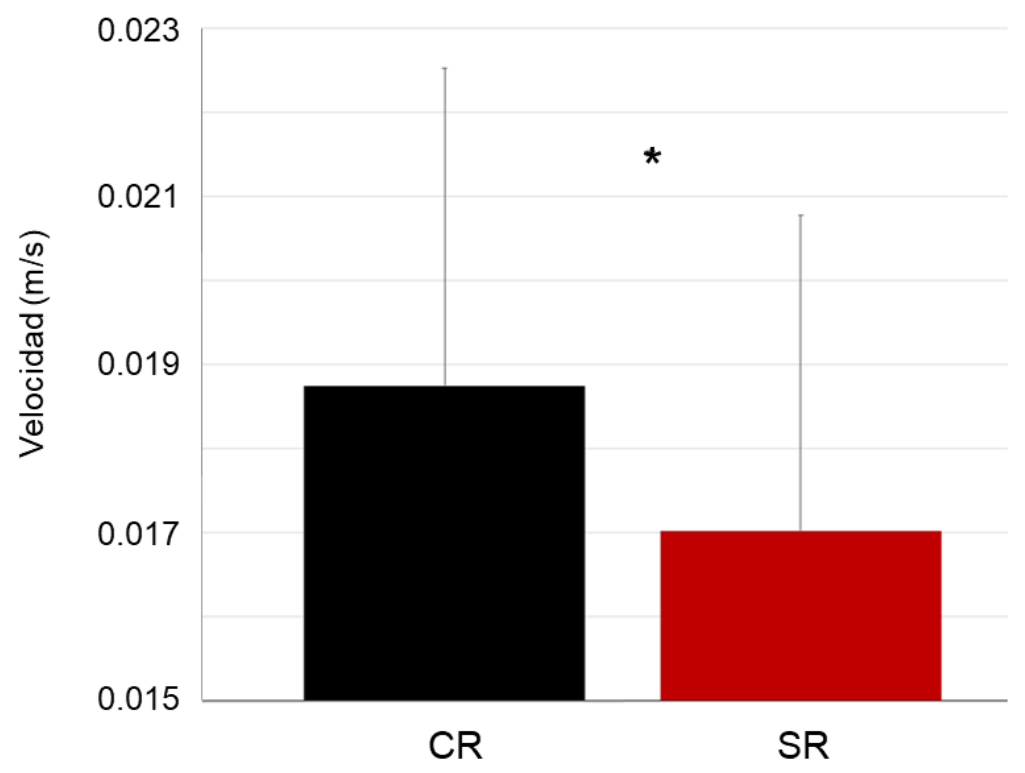

Figura 4 Velocidad en metros por segundo de la caminata de Coenobita compressus utilizando conchas remodeladas CR (barra negra) y sin remodelar SR (barra roja). El asterisco (*) señala diferencias significativas como resultado del análisis estadístico de comparación de muestras (prueba de $t$ de Student pareada; $\mathrm{P}=0.031$ ).

concha $(\mathrm{P}=0.0315)$, de modo que los cangrejos caminaron con una mayor velocidad utilizando conchas de gasterópodo remodeladas (Figura 4).

\section{Discusión y conclusiones}

Los resultados en este estudio mostraron que los cangrejos dejaron rastros sobre la arena con una menor proporción de huellas discontinuas al portar las conchas obtenidas directamente de los moluscos sin haber sido remodeladas por los ermitaños. Aunque este fenómeno no ha sido reportado anteriormente, Walker et al. (2003) publicaron rastros de Coenobita clypeatus Fabricius, 1787 que presentan huellas discontinuas como resultado de la caminata con una concha que probablemente ha sido remodelada por ser la que el cangrejo ocupaba originalmente al ser recolectado. Los cangrejos ermitaño caminan hacia el frente utilizando tres pares de apéndices; el primer par (quelípedos) es usado únicamente como soporte, mientras que la concha la sujetan utilizando los apéndices reducidos que se encuentran en su abdomen blando (Herreid y Full, 1986). En las conchas de Nerita scabricosta, que se utilizaron en este estudio, la remodelación representa la reducción en peso en un porcentaje de aproximadamente 10\% (datos no publicados, obtenidos del banco de conchas en el Laboratorio de Ecofisiología). El incremento de la proporción en los rastros continuos al usar conchas sin remodelar muestra que los ermitaños no sujetaron de manera continua una concha que es al menos $10 \%$ más pesada, arrastrándola en fricción con el sustrato de arena para desplazarse hacia el frente. El impacto de la concha sobre el sustrato también pudo mostrarse de acuerdo con las diferencias que se obtuvieron en este estudio en cuanto a la profundidad de la huella de las conchas en el rastro de los ermitaños. Estudios realizados en otros grupos de artrópodos han demostrado las ventajas de desplazarse cargando un menor peso, ya que los organismos tendrán un mejor desempeño en otras tareas tales como escapar de depredadores, búsqueda de alimento y exploración del ambiente (Torres-Contreras y Vásquez, 2004). 
Otro aspecto importante en la locomoción de organismos terrestres son las características del sustrato, donde el tipo y grosor del grano de arena, afectan directamente el movimiento del organismo en relación con la masa de carga (Urfi, 2016). Los resultados en este estudio mostraron que al arrastrar una concha que no ha sido remodelada (mayor masa sobre el sustrato), los cangrejos ermitaño redujeron la velocidad con la que pueden desplazarse, posiblemente debido a que requieren modificar el uso de energía para empujarse en el medio granular (Mazouchova et al., 2010; Chapple, 2012). Por lo tanto, el arrastre de una mayor área del organismo en el sustrato aumentará los costos de la locomoción debido a la resistencia causada por el mismo sustrato. De acuerdo con Li et al. (2013), la locomoción en un medio granular implica una compleja interacción a través de la presión y el ángulo del movimiento de los apéndices que los organismos utilizan para caminar, para lograr un estado parcialmente sólido del medio que les facilite la tracción.

\section{Referencias}

Abrams, P., 1978, Shell selection and utilization in a terrestrial hermit crab, Coenobita compressus (H. Milne Edwards): Oecologia, 34(2), 239-253.

Angel, J.E., 2000, Effects of shell fit on the biology of the hermit crab Pagurus longicarpus (Say): Journal of Experimental Marine Biology and Ecology, 243(2), 169-184.

Barnes, D., 1997, Ecology of tropical hermit crabs at Quirimba Island, Mozambique: a novel and locally important food source: Marine Ecology Progress Series, 161, 299-302.

Brown, D., 2008, Tracker (Java), Open Source Physics: California, E.U.A., publicado/ actualizado/revisado 2017, disponible en <http://www.opensourcephysics.org/items/ detail.cfm? $\mathrm{ID}=7365>$, consultado enero 2017.
Chapple, W., 2012, Kinematics of walking in the hermit crab, Pagurus pollicarus: Arthropod Structure \& Development, 41(2), 119-131.

Dana, J.D., 1851, Conspectus crustaceorum quae in orbis terrarum circumnavigatione, Carolo Wilkes e classe reipublicae foederatae duce, lexit et descripsit: Proceedings of the Academy of Natural Sciences of Philadelphia, 2, 267-272.

Davis, R.B., Minter, N.J., Braddy, S.J., 2007, The neoichnology of terrestrial arthropods: Palaeogeography, Palaeoclimatology, Palaeoecology, 255(3-4), 284-307.

de Wilde, P.A.W.J., 1973, On the ecology of Coenobita clypeatus in Curaçao with reference to reproduction, water economy and osmoregulation in terrestrial hermit crabs: Studies on the Fauna of Curaçao and Other Caribbean Islands, 44, 1-138.

Fabricius, F.C., 1787, Mantissa insectorum: sistens eorum species nuper detectas, adiectis characteribus genericis, differentiis specificis, emendationibus, observationibus: Copenhague, Dinamarca, Christ. Gottl. Proft., 348 p.

Guillén, F.G., Osorno, J.L., 1993, Elección de concha en Coenobita compressus (Decapoda: Coenobitidae): Revista de Biología Tropical, 41(1), 65-72.

Herreid, G.F., 1969, Integument permeability of crabs and adaptation to land: Comparative Biochemistry and Physiology, 29(1), 423-429.

Herreid, G.F., Full, R.J., 1986, Energetics of hermit crabs during locomotion: the cost of carrying a shell: Journal of Experimental Biology, 120, 297-308.

Instituto Nacional de Estadística y Geografía (INEGI), 2010, Mapa digital de los Estados Unidos Mexicanos Marco Geoestadístico Municipal: Ciudad de México, México, Instituto Nacional de Estadística y Geografía, disponible en <http://www.beta.inegi. org.mx/app/mapas/>, consultado 19 de octubre de 2017. 
Laidre, M.E., 2014, The social lives of hermits: Natural History, 122, 24-29.

Laidre, M.E., Patten, E., Pruitt, L., 2012, Costs of a more spacious home after remodeling by hermit crabs: Journal of The Royal Society Interface, 9(77), 3574-3577.

Lamarck, J.-B.P.A.M., 1815, Histoire naturelle des animaux sans vertèbres : présentant les caractères généraux et particuliers de ces animaux, leur distribution, leurs classes, leurs familles, leurs genres, et la citation des principales espèces qui s'y rapportent: précédée d'une introduction offrant la détermination des caractères essentiels de l'animal, sa distinction du végétal et des autres corps naturels, enfin, l'exposition des principes fondamentaux de la zoologie: París, Francia, Verdière, $496 \mathrm{p}$.

Leach, W.E., 1815, A tabular view of the external characters of four classes of animals which Linné arranged under Insecta; with the distribution of the genera composing three of these classes into orders and descriptions of several new genera and species: Series Transactions of the Linnean Society of London, 11, 306-400.

Li, C., Zhang, T., Goldman, D.I., 2013, A Terradynamics of Legged Locomotion on Granular Media: Science, 339(6126), 1408-1412.

Linnaeus, C., 1758, Systema Naturae per regna tria naturae, secundum classes, ordines, genera, species, cum characteribus, differentiis, synonymis, locis: Estocolmo, Suecia, Laurentii Salvii, 824 p.
Mazouchova, N., Gravish, N., Savu, A., Goldman, D.I., 2010, Utilization of granular solidification during terrestrial locomotion of hatchling sea turtles: Biology Letters, 6(3), 398-401.

Milne-Edwards, H., 1834, Histoire Naturelle des Crustacés, Comprenant L'anatomie, La Physiologie et la Classification de ces Animaux: París, Francia, Librairie Encyclopédique de Roret, 532 p.

Osorno, J., Fernández, L., Rodríguez, C., 1998, Are hermit crabs looking for light and large shells?: evidence from natural and field induced shell exchanges: Journal of Experimental Marine Biology and Ecology, 222(1), 163-173.

Torres-Contreras, H., Vásquez, R.A., 2004, A field experiment on the influence of load transportation and patch distance on the locomotion velocity of Dorymyrmex goetschi (Hymenoptera, Formicidae): Insect Sociaux, 51(3), 265-270.

Urfi, A.J., 2016, Animal locomotion in different mediums. The Adaptations of Wetland Organisms: Resonance, 21(6), 545-556.

Vermeij, G.J., 2012, Evolution: Remodeling Hermit Shellters: Current Biology, 22(20), R882-R884.

Walker, S.E., Holland, S.M., Gardiner, L., 2003, Coenobichnus currani (new ichnogenus and ichnospecies): fossil trackway of a land hermit crab, Early Holocene, San Salvador, Bahamas: Journal of Paleontology, 77(3), 576-582. 